wartung konkreter Vorteile koordinieren (S. 147). Sie zeichnen sich nicht durch die starke Formalisierung von Regeln und Mitgliedschaftsbedingungen aus, wie dies Verbände tun.

Der Fokus der Studie Barthels liegt eindeutig im Bereich einer theoretischen Konzeptionalisierung. Häufig leiden zu Büchern umgearbeitete Dissertationen an einer Theorielastigkeit, die viele Seiten füllt und den praxisorientierten Leser oft schon nach kurzer Zeit langweilen. Hier liegt der Fall anders. In der Kürze (und in der sprachlichen Klarheit) liegt die Würze. Auch wenn empirische Sachverhalte einen eher veranschaulichenden Charakter haben und nicht im Rahmen von Fallstudien dargestellt sind, büßt die Studie nicht an Nützlichkeit und Anregungen für den interessierten Praktiker ein, zumal dieser noch zusätzlich auf die nach wie vor sehr lesenswerte ausführliche empirische Studie zur entwicklungsbegleitenden Normung von Jochen Barthel und Bernd Steffensen aus dem Jahre 2000 (,Koordination im Innovationsprozess", erschienen im NomosVerlag) zurückgreifen kann.

Alles in allem gibt die Studie zahlreiche Anregungen für eine entwicklungsbegleitende Standardisierung. So der Hinweis: „Dabei wäre z. B. die Förderung der entwicklungsbegleitenden Vereinbarung von Standards für Kompatibilität in Verbänden nicht optimal. Erfolgversprechender sind Initiativen in Verbänden, sich auf die eigenen Stärken $\mathrm{zu}$ besinnen und $\mathrm{zu}$ versuchen, Brücken zwischen verschiedenen Wegen der Standardisierung zu schlagen" (S. 174). Es bleibt zu hoffen, dass sich an dieses inspirierende Buch vertiefende empirische Studien und Diskurse anschließen, die z. B. Erfahrungen des Auslandes und auf europäischer Ebene stärker diskutieren und Antworten auf die Frage liefern, was sich in Deutschland daraus lernen lässt und welche Initiativen in Bezug auf den Standort Deutschland notwendig werden.

\section{KURZVORSTELLUNGEN VON BÜCHERN}

\author{
ULRICH DOLATA: Unternehmen Tech- \\ nik. Akteure, Interaktionsmuster und \\ strukturelle Kontexte der Technikent- \\ wicklung: Ein Theorierahmen. Berlin: \\ edition sigma, 2002. 333 S., 24,90€, \\ ISBN 3-89404-500-0
}

Wie entsteht Technik? Wer macht sie, und unter welchen Umständen geschieht das?

Der Autor entfaltet in diesem Buch einen theoretischen Leitfaden zur Analyse der sozialen Genese, Aneignung und Regulierung neuer Techniken - ein Konzept jenseits deterministischer Engführungen und sozialkonstruktivistischer Beliebigkeit, das am Beispiel der Gentechnik einem Plausibilitätstest unterzogen wird.

Dolatas Analyserahmen führt drei Aspekte zu einer integrativen Perspektive zusammen: das technikbezogene Handeln der involvierten Akteure, die durch sie ausgeprägten kooperativen bzw. kompetitiven Interaktionsmuster sowie die technischen, sozioökonomischen und kulturellen Kontexte, die ihr Handeln prägen. Ein umfassendes Verständnis von Technikgeneseprozessen erfordert, sehr unterschiedliche Dimensionen in ihrer Wechselwirkung zu erfassen: das Verhältnis von Organisationen, Personen und nichtorganisierten Kollektiven; den Stellenwert von Macht und Vertrauen in industriellen Kooperations- und politischen Aushandlungssystemen sowie das Spannungsverhältnis von Vernetzung und Konkurrenz; die handlungsstrukturierenden Wirkungen des jeweiligen Typs von Technik; dessen Einbindung in Innovationssysteme und Technikstile und vieles anderes mehr.

(Verlagsankündigung) 
SIMON JOSS, SERGIO BELLUCCI (Eds.): Participatory Technology Assessment. European Perspectives. London: Centre for the Study of Democracy, 2002. 320 S., £ 15.00, ISBN 0-85374-803-9

Since the early 1990s, an increasing number of citizens, stakeholders and user groups have become involved in assessing new scientific and technological developments. This involvement has taken various forms, including citizens' panels, scenario workshops, round tables and consensus conferences. The aim of such "participatory technology assessment" is to provide advice to policymakers and to encourage wider public debate about sociotechnological developments.

This volume gives a comprehensive overview of recent developments in participatory technology assessment in a variety of European national and institutional contexts. It includes a research framework that provides a basis for both theoretical and practical analysis; contains studies of 16 participatory initiatives in Austria, Denmark, Germany, the Netherlands, Switzerland and the United Kingdom; and offers in-depth, cross-country comparisons focusing on important issues such as the methodological design, political role and impact of participatory technology assessment.

This book is based on a two-year European Commission funded research project (EUROPTA).

(Verlagsankündigung)

$\gg$

\section{ANGELIKA SAUPE: Verlebendigung der Technik - Perspektiven im feministi- schen Technikdiskurs. Bielefeld: Kleine Verlag GmbH, 2002 (Wissenschaftliche Reihe Band 141). 360 S., 24,05€, ISBN 3-89370-364-0}

Kunst-Naturen werden in spezifischer Weise durch das technowissenschaftliche Wissen produziert - sowohl materiell wie diskursiv. Besonders der Begriff des Lebens unterliegt dabei einer konstitutiven Wendung: „Leben bedeutet Information... Wer auch immer es kreiert, muss im Zeitalter des Codes weder auf Anfang und Ende, auf Genealogie und Herkunft, noch auf Irdisches und Transzendenz rekurrieren" (Lisbeth Trallori, 1996). So werden die herkömmlichen Dualismen wie Natur und Kultur, Lebendiges und Technisches, Mensch und Maschine etc. neu verortet.

Vor diesem Hintergrund analysiert die vorliegende Arbeit den deutschsprachigen feministischen Technikdiskurs. Ihr Fokus richtet sich auf die Rekonstruktion der theoretischen Prämissen der verschiedenen Ansätze, die das Verhältnis von Technik und Geschlecht gesellschaftstheoretisch zu bestimmen suchen. Die Autorin kritisiert, dass der feministische Technikdiskurs überwiegend in einer Tradition zivilisations- und entfremdungskritisch argumentierender Kulturkritik verharrt. Mit dem Ansatz der US-amerikanischen Wissenschaftsforscherin Donna Haraway sowie einem Rückgriff auf die Gesellschaftstheorie Alfred SohnRethels wird diese Argumentationslinie aufgebrochen.

Der Fokus der Arbeit richtet sich auf eine dynamische Kritik grundlegender Muster technologischer Selbstrepräsentation von Gesellschaft. Dabei wird die These vertreten, dass die fortschreitende Technisierung von Natur kein universelles Verschwinden bzw. Zerstören von Natur und Leben bedeutet, sondern komplementär eine Tendenz zur „Verlebendigung der Technik“ die Umstrukturierung sozioökonomischer Basiskonstellationen begleitet.

Die Arbeit erhebt den Anspruch, den feministischen Technikdiskurs seit den 1970er Jahren umfassend zu reflektieren und in neue erkenntniskritische Perspektiven zu überführen.

(Verlagsankündigung)

BARBARA SKORUPINSKI, KLAUS OTT: Partizipative TA als ethisches Erfordernis. Warum das Urteil der Bürger unverzichtbar ist. Arbeitsdokument des Zentrum TA-SWISS, 2002 (TA-DT 31/2002)

Das Forschungsprojekt „Technikfolgenabschätzung und Ethik" wurde vom Schweizerischen Nationalfonds im Schwerpunktprogramm Bio 
technologie von März 1995 bis Februar 2000 gefördert. Es war unter der Leitung von Dr. Barbara Skorupinski am Institut für Sozialethik der Universität Zürich angesiedelt. Mitarbeit im Projekt und Kooperationspartner war Prof. Dr. Konrad Ott, Professur für Umweltethik, ErnstMoritz-Arndt-Universität Greifswald. Das interdisziplinäre Forschungsprojekt hatte sich eine systematische Analyse und Klärung des Verhältnisses von TA und Ethik zum Ziel gesetzt.

Eine ausführliche Darstellung des Projektes von Barbara Skorupinski ist in den TADatenbank-Nachrichten, Heft 2, 9. Jahrgang, Juni 2000, S. 88-93 enthalten (http://www.itas. fzk.de/deu/tadn/tadn002/skor00a.htm).

Der Projektbericht wurde im vdf-Verlag, Zürich 2000, veröffentlicht. Diese Publikation ist jetzt populärwissenschaftlich zusammengefasst als Arbeitsdokument unter dem oben angegebenen Titel kostenlos erhältlich von: Zentrum TA-SWISS, Birkenweg 61, CH-3003 Bern, per Fax +41 (0) 31 / 3233659 .

।

HANS-ULRICH ZABEL (Hrsg.): Betriebliches Umweltmanagement - nachhaltig und interdisziplinär. München: Erich Schmidt Verlag, 2002 (Reihe: Initiativen zum Umweltschutz, Band 46), 427 S., 56,00 €, ISBN 3-503-07007-9

Angesichts wachsender sozialer und ökologischer Probleme mit zunehmend globalen Bedrohungsrisiken zeigt die Nachhaltigkeitsdiskussion Bemühungen um die Rückgewinnung des menschlichen Maßes in den Wirtschaftsund Lebensbeziehungen der Menschen. Die Orientierung auf das Leitbild der Nachhaltigkeit ist eine sehr facettenreiche und komplexe Herauforderung, da eine Vielzahl von Wissenschaftsdisziplinen und vor allem deren Schnittstellen betroffen sind.

Die Herausforderung an die ,scientific community" lautet: Konsequent humanistische Zielausrichtung auf dem Weg interdisziplinärer Arbeitsweise. Das Betriebliche Umweltmanagement erhält dabei eine Schlüsselfunktion.

Die in diesem neuen Band enthaltenen Beiträge dokumentieren die Ergebnisse einer dieser Herausforderung verpflichteten Tagung, die im März 2001 vom Lehrstuhl „BWL - insbesondere Betriebliches Umweltmanagement" an der Martin-Luther-Universität Halle stattfand. Die zentralen Themen sind durch die Stichworte Verhaltensaspekte, Umweltmanagementsysteme, Kreislaufwirtschaft und Informationsgesellschaft gekennzeichnet.

(Verlagsankündigung)

GOTTHARD STEIN (Hrsg.): Umwelt und Technik im Gleichklang. Technikfolgenforschung und Systemanalyse in Deutschland. Berlin u. a.: SpringerVerlag, 2003. 408 S., 49,95 €, ISBN 3-540-43872-6

Seit Anfang der 80er Jahre existiert im Rahmen der Helmholtz-Gemeinschaft (HGF) der Ausschuss „Systemanalyse und Technikfolgenabschätzung“". Seine Aktivitäten beschränken sich aber nicht nur auf die HGF, sondern er hat als Mitglieder auch andere deutsche Institutionen, die auf dem Feld der Systemanalyse und der Technikfolgen forschen.

Die Neustrukturierung der HGF gibt der Systemanalyse und Technikfolgenabschätzung neue Bedeutung und Impulse als wichtige interdisziplinäre Querschnittsaktivität. Im derzeitigen frühen Stadium der ,neuen“ HGF ist der Bereich „Nachhaltiges Wirtschaften“ ein vorläufiger Heimathafen für die vielfältigen Aktivitäten der Systemanalyse und Technikfolgenabschätzung. Diese Einordnung kann allerdings nur als Einstieg verstanden werden, weil sie in keiner Weise der multidimensionalen Struktur der Forschung zur Systemanalyse und der Technikfolgen gerecht wird. Der vorliegende Sammelband kann und soll auch nicht Systemanalyse und Technikfolgenabschätzung aus einem Guss darstellen, sondern bewusst die Vielfältigkeit und Komplexität und damit Querschnittsfunktion und Politikberatung als wesentliche Aufgabenstellung verdeutlichen.

Die in diesem Buch behandelten Themen reichen von Beiträgen zur Konzeption interdisziplinärer Technikfolgenforschung über nachhaltige Entwicklung, Mobilität, Stoffstromanalyse bis zur Risikobewertung und - 
kommunikation. Weiterhin gibt es Ausarbeitungen zur Technikfolgenabschätzung in unterschiedlichen Technikfeldern.

Die Arbeiten geben einen Einblick in Breite und Vielfältigkeit der Analysen, ihrer Methoden und Modelle. Sie sollen auch einen Eindruck aus der Werkstatt der HGF-Institute und anderer deutscher Institutionen geben und damit Potenzial, Ausrichtung und Schwerpunkte der deutschen Forschung in diesem wichtigen Feld illustrieren.

(aus dem Vorwort)

》)

FRIEDER RUBIK: Integrierte Produktpolitik. Marburg: metropolis Verlag, 2002. 426 S., 32,80 €, ISBN 3-89518-387-3

Produktpolitische Fragestellungen haben in der Umweltpolitik an Bedeutung gewonnen. „Integrierte Produktpolitik“ (IPP) möchte die produktbezogenen Aktivitäten der verschiedenen Akteure auf der Angebots- und Nachfrageseite in ökologischer Absicht stimulieren.

Die Veröffentlichung nimmt eine Einordnung der Produktpolitik in verschiedene wissenschaftliche Disziplinen vor und entwickelt ein vierstufiges Entwicklungsmodell ihrer Implementation. Illustriert und empirisch unterlegt wird dieses Modell anhand der Produktpolitiken in fünf Ländern (Dänemark, Deutschland, Großbritannien, Niederland und Schweden), wobei produktpolitische Überlegungen seitens der EU ergänzend eingeführt werden.

Ausgehend vom Entwicklungsmodell werden elf Anforderungen an eine IPP-Konzeption erarbeitet, wobei insbesondere dem Aspekt der Markttransformation durch Umweltinnovationen eine besondere Bedeutung beigemessen wird. Die Anforderungen bilden den Ausgangspunkt für die Entwicklung einer deutschen IPPKonzeption, in der auf Ziele, Rolle der Akteure, Grundsätze, inhaltliche Schwerpunkte und Instrumente eingegangen wird.

Empirisch unterlegt wird die Arbeit anhand zweier Fallbeispiele (Kühlgeräte und Holzmöbel), in denen das Zusammenwirken zwischen Staat und anderen Akteuren nachge- zeichnet wird. Dabei zeigen sich bemerkenswerte Differenzen zwischen beiden Beispielen.

Die Arbeit schließt mit Empfehlungen für produktgruppenübergreifende IPP-Aktivitäten, wie etwa Pilotprojekte als Beispiele einer kooperativen Produktpolitik, sowie über fünfzig ausführlich dargelegte Maßnahmen.

(Verlagsankündigung)

$\ll$

MARTIN KAIMER, DIETHARD SCHADE: Zukunftsfähige Hausmüllentsorgung: Effiziente Kreislaufwirtschaft durch Entlastung der Bürger. Berlin, Bielefeld, München: Erich Schmidt Verlag, 2002 (Reihe: Abfallwirtschaft in Forschung und Praxis, Band 126), 170 S., 26,80 $€$, ISBN 3-503-07021-4

Die Abfallentsorgung steht derzeit vor einem Dilemma, weil trotz großer Anstrengungen zur Trennung der verschiedenen Abfallbestandteile und der damit verbundenen hohen Belastungen der Haushalte die Ziele einer möglichst sortenreinen Erfassung von Wertstoffen und einer zuverlässigen Aussonderung von Schadstoffen nicht erreicht werden. Noch höhere Anforderungen an die Getrenntsammlung werden dieses Problem nicht lösen, sondern vor allem die Haushalte mit zusätzlichen Aufgaben und Kosten belasten.

Die Autoren zeigen in dieser Studie auf, wie die zentralen Ziele der Kreislaufwirtschaft auch bei geringerem Trennaufwand für Haushalte erreicht werden können. Mit neuen leistungsfähigen automatischen Sortiertechniken und neuen Techniken für die Abfallbehandlung wird es möglich, alle im Abfall enthaltenen Wertstoffe weitgehend $\mathrm{zu}$ nutzen, die nicht verwertbaren Abfallbestandteile umweltgerecht $\mathrm{zu}$ beseitigen und eine Abfallwirtschaft ohne (oberirdische) Deponie zu erreichen. Diese Ziele sind nicht kurzfristig zu erreichen, es müssen aber bereits jetzt Maßnahmen zur Schaffung der entsprechenden Rahmenbedingungen in die Wege geleitet werden.

(Verlagsankündigung) 